\title{
Cognitive Decline in Korean Patients with Neurocognitive Disorder due to Traumatic Brain Injury: A Control for Premorbid Intelligence
}

\author{
Kyu-Sic Hwang ${ }^{1,2}$, Seung-Ho Jang ${ }^{1,2,3}$, Min-Jung Soh ${ }^{1,2}$, Hye-Jin Lee ${ }^{4}$, and Sang-Yeol Lee ${ }^{1,2,3 凶}$ \\ ${ }^{1}$ Department of Psychiatry, Wonkwang University Hospital, Iksan, Republic of Korea \\ ${ }^{2}$ Korea Brain-Behavior Mental Health Institute, Iksan, Republic of Korea \\ ${ }^{3}$ Department of Psychiatry, School of Medicine, Wonkwang University, Iksan, Republic of Korea \\ ${ }^{4}$ Department of Public Health, Wonkwang University Graduate School, Iksan, Republic of Korea
}

\begin{abstract}
Objective Previous studies of cognitive decline in patients with neurocognitive disorder due to traumatic brain injury (NCD-TBI) have often failed to control for baseline factors such as premorbid intelligence. The purpose of the current study was to estimate and compare cognitive function among three groups (controls, complicated mild/moderate TBI, and severe TBI) after controlling for premorbid intelligence.

Methods Severity of TBI was classified as complicated mild/moderate or severe based on duration of loss of consciousness and brain neuroimaging results. Premorbid intelligence quotients (IQs) were estimated with the Oklahoma Premorbid Intelligence Estimate. There were no differences in premorbid intelligence between the groups, which were also matched for age and education. Current cognitive function was evaluated with the Wechsler Adult Intelligence Scale-Fourth Edition.

Results Comparison of current cognitive function among the three groups indicated significant group differences for all indexes and subtest scores. Processing speed showed the highest effect size. However, only working memory differed significantly between the two NCD-TBI groups.

Conclusion The present findings suggest that mental memory manipulation processes seem to be more sensitive to TBI severity than are perceptual-motor processes. Specifically, both auditory rehearsal/discrimination and mental alertness/manipulation will be most strongly influenced by TBI severity.

Psychiatry Investig 2019;16(12):889-895
\end{abstract}

Key Words Neurocognitive disorder, Traumatic brain injury, Cognitive decline, Wechsler adult intelligence scale-fourth edition, Premorbid intelligence.

\section{INTRODUCTION}

Neurocognitive disorder due to traumatic brain injury (NCDTBI) is often accompanied by cognitive decline. ${ }^{1,2}$ According to the Diagnostic and Statistical Manual of Mental DisordersFifth Edition (DSM-5), a diverse range of neurocognitive domains are affected in patients with NCD-TBI. Research indicates impairments of memory, as well as of moral judgment

Received: May 20, 2019 Revised: June 18, 2019

Accepted: July 30, 2019

$\triangle$ Correspondence: Sang-Yeol Lee, MD, PhD

Department of Psychiatry, School of Medicine, Wonkwang University, 895 Muwang-ro, Iksan 54538, Republic of Korea

Tel: +82-63-859-1044, Fax: +82-63-857-1043, E-mail: psysangyeol@hanmail.net

(a) This is an Open Access article distributed under the terms of the Creative Commons Attribution Non-Commercial License (https://creativecommons.org/licenses/by$\mathrm{nc} / 4.0$ ) which permits unrestricted non-commercial use, distribution, and reproduction in any medium, provided the original work is properly cited. and social cognition in NCD-TBI. ${ }^{1,3,4}$

Here, current cognitive function is assessed with the Wechsler Adult Intelligence Scale-Fourth Edition (WAIS-IV). ${ }^{5}$ The WAIS is a test of neurocognitive function that measures an individual's overall intellectual ability, crystallized and fluid intelligence, short-term and working memory, and processing speed. ${ }^{6} \mathrm{~A}$ recent study investigated cognitive function in patients with NCD-TBI with the WAIS-IV, reporting significant differences for all indexes and subtest scores in patients with severe TBI compared with controls. ${ }^{7}$ Moreover, there were significant differences in working memory, processing speed, and full-scale IQ between patients with complicated mild/moderate TBI and controls. ${ }^{7}$ A significant difference in processing speed between patients with complicated mild/moderate TBI and those with severe TBI has also been reported. ${ }^{8}$ These results indicate that there are differences in the domains affected and the extent of 
damage depending on the severity of TBI.

Although previous studies have consistently highlighted differences in WAIS between patients with differing severities of NCD-TBI, as well as between patients with NCD-TBI and controls, no consideration has been given to premorbid intelligence. ${ }^{7,8}$ For example, there may be differing degrees of damage for two people with premorbid IQs of 125 and 85, which both drop to 75 after TBI, because when considering premorbid IQ, their scores have changed by 50 and 10 points, respectively. Therefore, it is imperative to consider premorbid IQ when analyzing data pertaining to retention of cognitive function after complicated TBI.

As this suggests, neuropsychological assessment to determine cognitive functioning before and after NCD-TBI onset is essential to fully understand the effects of brain damage. ${ }^{9}$ Indeed, assessing a patient's premorbid cognitive function is a crucial first step for determining current cognitive function, predicting future progress, and establishing therapeutic interventions. ${ }^{10}$ In particular, it is crucial to determine the degree of current cognitive impairment within specific cognitive domains compared with premorbid cognitive function. ${ }^{11}$ Therefore, developing an assessment tool to determine the degree of current cognitive impairment and to estimate the level of premorbid cognitive function is important. ${ }^{12}$

The current study was based on the hypothesis that there are no significant differences in premorbid intelligence among three groups: controls, patients with complicated mild/moderate TBI, and patients with severe TBI. We used the recently developed Oklahoma Premorbid Intelligence Estimate (OPIE) to estimate premorbid intelligence. ${ }^{11,13}$ Subsequently, we analyzed differences in cognitive function indexes and subtest scores among the three groups and compared the results with previous studies.

\section{METHODS}

\section{Design and participants}

Initially, 103 Korean patients with NCD-TBI were enrolled in the study. They all consisted of inpatients and outpatients who were receiving care in the department of psychiatry. TBI groups were classified based on severity of trauma, which was determined by duration of loss of consciousness and abnormal neuroimaging results. ${ }^{1,2}$ Patients with complicated mild and moderate TBI were combined into a single group because previous studies have indicated that long-term findings for such patients are similar. ${ }^{7,14}$ Consequently, there were two TBI groups: complicated mild/moderate TBI and severe TBI.

To control for the effects of age and education level on premorbid intelligence, participants in the control and severe TBI groups were matched for these variables with participants in the complicated mild/moderate TBI group. Data for healthy controls comprised partial data from the standardization study of the Korean WAIS-IV. ${ }^{15}$ Finally, 94 participants were included in the study (controls, $\mathrm{n}=32$; complicated mild/moderate TBI, $\mathrm{n}=32$; severe TBI group, $\mathrm{n}=31$ ).

All the study procedures were reviewed and approved by the Local Institutional Ethics Review Board (WKUH 201704-023). All the participants were given informed consent before study.

\section{Measures}

\section{Wechsler adult intelligence scale-fourth edition}

The Korean Wechsler adult intelligence scale-fourth edition (WAIS-IV) was used to assess current cognitive function of patients with NCD-TBI. ${ }^{16}$ The Korean WAIS-IV was standardized in 2012, showing good internal consistency ( 0.87 to 0.97 ) and test-retest reliability ( 0.72 to 0.89$).{ }^{15}$ Construct validity was confirmed with the hierarchical four-factor model. Concurrent validity with the Korean Wechsler Intelligence Scale for Children-Fourth Edition was good at $\mathrm{r}=0.80$.

\section{Oklahoma premorbid intelligence estimate}

To account for premorbid intelligence, premorbid IQ of TBI patients was estimated. The Oklahoma premorbid intelligence estimate (OPIE) method was recently developed and has been validated in clinical settings. The OPIE includes regression formulas developed by combining demographic variables and WAIS subtest scores. ${ }^{17-19}$

In the present study, premorbid intelligence was estimated with the Korean version of the OPIE method for the WAIS-IV (OPIE-IV). ${ }^{13,20}$ The regression formulas of the Korean OPIE-IV comprise five types of algorithms, one of which uses three predictive variables (i.e., WAIS-IV information subtest, age, and education level). Previous application of the premorbid intelligence estimation formula to data from brain injury cases provided the rationale for applying the selected algorithm in the present study. ${ }^{18-20}$

The full formulas used in the current study were as follows: $[67.173+1.722 \times($ information subtest raw score $)+0.161 \times($ age $)+$ $0.995 \times$ (education level)]. Education level was scored as 1 for $<8$ years, 2 for $9-11$ years, 3 for 12 years, 4 for $13-15$ years, and 5 for $\geq 16$ years.

\section{Statistical analyses}

One-way ANOVA was conducted to examine differences in demographic variables among the groups. Furthermore, frequency analysis of the descriptive classification for both premorbid and current IQ was also performed. Subsequently, a paired t-test was used to identify any difference between esti- 
mated premorbid and current intelligence within groups. Effect sizes (Cohen's d) were calculated for statistically significant differences. ${ }^{21}$ Next, one-way ANOVA was performed among the groups to identify differences among scores for each index and subtest scores based on current intelligence. For statistically significant differences, effect sizes (partial eta squared, $\left.\eta^{2}\right)$ were calculated.

\section{RESULTS}

\section{Participant baseline characteristics}

Table 1 presents demographic data and premorbid IQs for included participants. No significant differences were observed among the groups $[F(2,92)=0.665, p=0.517]$. Similarly, there were no significant differences among the groups for educational level $[\mathrm{F}(2,92)=0.199, \mathrm{p}=0.082]$ or estimated premorbid IQ $[F(2,92)=0.171, p=0.843]$.

\section{Premorbid and current full-scale IQ}

Table 2 presents results for the frequency analysis of groupbased descriptive classification. Based on estimated premorbid IQ, there were no participants in any group that fell within the ratings of "Very superior," "Superior," or "Extremely low." Moreover, more than $85 \%$ of each group were "Average" and "Low average."

Table 1. Participant baseline characteristics

\begin{tabular}{|c|c|c|c|c|c|}
\hline Variable & $\begin{array}{c}\text { Normal control group }(\mathrm{N}=32) \\
\text { Mean } \pm \mathrm{SD}\end{array}$ & $\begin{array}{c}\text { Complicated mild/ } \\
\text { moderate TBI group }(\mathrm{N}=32) \\
\text { Mean } \pm \mathrm{SD}\end{array}$ & $\begin{array}{l}\text { Severe TBI group }(\mathrm{N}=31) \\
\text { Mean } \pm \mathrm{SD}\end{array}$ & $\mathrm{F}$ & $\mathrm{p}$ \\
\hline Age & $49.15 \pm 13.28$ & $52.43 \pm 12.84$ & $49.13 \pm 13.36$ & 0.665 & 0.517 \\
\hline Years of education & $9.66 \pm 3.06$ & $9.88 \pm 2.93$ & $10.16 \pm 3.55$ & 0.199 & 0.820 \\
\hline Estimated premorbid IQ & $89.52 \pm 8.56$ & $89.14 \pm 8.35$ & $88.35 \pm 7.15$ & 0.171 & 0.843 \\
\hline
\end{tabular}

IQ: intelligence quotient, TBI: traumatic brain injury

Table 2. Frequency analysis of premorbid and current full-scale $I Q$, by group

\begin{tabular}{|c|c|c|c|c|c|c|}
\hline \multirow{2}{*}{$\begin{array}{c}\text { Descriptive } \\
\text { classification* }\end{array}$} & \multicolumn{2}{|c|}{ Normal control group $(\mathrm{N}=32)$} & \multicolumn{2}{|c|}{$\begin{array}{l}\text { Complicated mild/ } \\
\text { moderate TBI group }(\mathrm{N}=32)\end{array}$} & \multicolumn{2}{|c|}{ Severe TBI group $(\mathrm{N}=31)$} \\
\hline & $\begin{array}{c}\text { Estimated premorbid IQ, } \\
\mathrm{N}(\%) \\
\end{array}$ & $\begin{array}{c}\text { Current IQ, } \\
\mathrm{N}(\%)\end{array}$ & $\begin{array}{c}\text { Estimated premorbid IQ, } \\
\mathrm{N}(\%) \\
\end{array}$ & $\begin{array}{c}\text { Current IQ, } \\
\mathrm{N}(\%)\end{array}$ & $\begin{array}{c}\text { Estimated premorbid IQ, } \\
\mathrm{N}(\%) \\
\end{array}$ & $\begin{array}{c}\text { Current IQ, } \\
\mathrm{N}(\%)\end{array}$ \\
\hline Very superior & $0(0)$ & $0(0)$ & $0(0)$ & $0(0)$ & $0(0)$ & $0(0)$ \\
\hline Superior & $0(0)$ & $0(0)$ & $0(0)$ & $0(0)$ & $0(0)$ & $0(0)$ \\
\hline High average & $1(3.1)$ & $3(9.4)$ & $1(3.1)$ & $0(0)$ & $0(0)$ & $0(0)$ \\
\hline Average & $14(43.8)$ & $21(65.6)$ & $13(40.6)$ & $4(12.5)$ & $14(45.2)$ & $2(6.5)$ \\
\hline Low average & $15(46.9)$ & $3(9.4)$ & $14(43.8)$ & $6(18.8)$ & $13(41.9)$ & $3(9.7)$ \\
\hline Borderline & $2(6.3)$ & $5(15.6)$ & $4(12.5)$ & $9(28.1)$ & $4(12.9)$ & $5(16.1)$ \\
\hline Extremely low & $0(0)$ & $0(0)$ & $0(0)$ & $13(40.6)$ & $0(0)$ & $21(67.7)$ \\
\hline
\end{tabular}

*the classification is followed by descriptive classification of intelligence by Wechsler, which refers "Very superior" for 130 and above, "Superior" for 120 to 129, "High average" for 110 to 119, "Average" for 90 to 109, "Low average" for 80 to 89, "Borderline" for 70 to 79, and "Extremely low" for 69 and below. IQ: intelligence quotient, TBI: traumatic brain injury
However, based on current IQ, 32\% of patients in the complicated mild/moderate TBI group had "Average" and "Low average" ratings, while only $17 \%$ of the severe TBI group had "Average" and "Low average" ratings. In contrast, current full-scale IQ for controls suggested that more than $84 \%$ had "Average" and "Low average" ratings. Finally, "Extremely low" ratings accounted for $0 \%, 40.6 \%$, and $67.7 \%$ of the control, complicated mild/moderate TBI, and severe TBI groups, respectively.

\section{Within group differences between premorbid and current full-scale IQ}

Table 3 presents differences between estimated premorbid and current IQ within each group. There was no significant difference between estimated premorbid and current IQ for controls (89.52 vs. 94.72 , respectively; difference: $-5.20 ; \mathrm{t}(31)=-1.848$, $\mathrm{p}=0.074$ ). In contrast, there was a significant difference between estimated premorbid and current IQ in the complicated mild/ moderate TBI group ( 89.14 vs. 70.53 , respectively; difference: $18.61 \mathrm{pts} ; \mathrm{t}(31)=8.61, \mathrm{p}<0.001)$, with the difference corresponding to a "large" effect size $(\mathrm{d}=1.44)$. In the severe TBI group, there was also a statistically significant difference between estimated premorbid and current IQ ( 88.35 vs. 62.65 , respectively; difference: $25.70 ; \mathrm{t}(30)=12.67, \mathrm{p}<0.001)$ and this also represented a "large" effect size $(\mathrm{d}=2.27)$. 


\section{Differences in current cognition}

Table 4 shows the results of a one-way ANOVA comparing current scores for each index and subtest among the groups. All the indexes showed statistically significant group differences with "large" effect sizes. In particular, processing speed and cognitive proficiency accounted for over $50 \%$ variance $(58.5 \%$ and $54.9 \%$, respectively).

Based on scaled subtest scores, there were significant group differences with "large" effect sizes for all subtests except matrix reasoning. Moreover, symbol search and coding subtests accounted for over $50 \%$ of the variance $(59.6 \%$ and $52.9 \%$, respectively).

Table 4 also presents results of the post-hoc test between groups. Comparing complicated mild/moderate TBI with severe TBI, there was a significant difference in working memory, including its subtests (symbol search and coding). How-

Table 3. Within group differences between premorbid and current full-scale IQ

\begin{tabular}{|c|c|c|c|c|c|c|}
\hline Group & $\begin{array}{c}\text { Estimated premorbid IQ } \\
\text { Mean } \pm \text { SD }\end{array}$ & $\begin{array}{l}\text { Current IQ } \\
\text { Mean } \pm \text { SD }\end{array}$ & Difference value & $\mathrm{t}$ & $\mathrm{p}$ & $\mathrm{d}$ \\
\hline Normal control group $(\mathrm{N}=32)$ & $89.52 \pm 8.56$ & $94.72 \pm 11.89$ & -5.20 & -1.85 & 0.074 & NS \\
\hline $\begin{array}{l}\text { Complicated mild/ } \\
\text { moderate TBI group }(\mathrm{N}=32)\end{array}$ & $89.14 \pm 8.35$ & $70.53 \pm 17.48$ & 18.61 & $8.61^{*}$ & 0.000 & 1.44 \\
\hline Severe TBI group $(\mathrm{N}=31)$ & $88.35 \pm 7.15$ & $62.65 \pm 15.87$ & 25.70 & $12.67^{*}$ & 0.000 & 2.27 \\
\hline
\end{tabular}

${ }^{*} \mathrm{p}<0.001$. IQ: intelligence quotient, TBI: traumatic brain injury, NA: not significant

Table 4. Differences in current cognition, by group

\begin{tabular}{|c|c|c|c|c|c|c|c|}
\hline \multicolumn{2}{|c|}{ Current score } & \multicolumn{3}{|c|}{ Group } & \multicolumn{3}{|c|}{ Statistic } \\
\hline Index & Subtest & $\begin{array}{c}\text { Normal control }(\mathrm{N}=32) \\
\text { Mean } \pm \mathrm{SD}\end{array}$ & $\begin{array}{c}\text { Complicated mild/ } \\
\text { moderate TBI }(\mathrm{N}=32) \\
\text { Mean } \pm \mathrm{SD}\end{array}$ & $\begin{array}{c}\text { Severe TBI group }(\mathrm{N}=31) \\
\text { Mean } \pm \mathrm{SD}\end{array}$ & $\mathrm{F}$ & $\mathrm{p}$ & $\eta^{2}$ \\
\hline \multirow[t]{4}{*}{$\overline{\mathrm{VCI}}$} & & $96.50 \pm 12.86$ & $78.53 \pm 17.18$ & $72.74 \pm 15.66$ & $20.67^{\ddagger}$ & 0.000 & 0.310 \\
\hline & SI & $8.78 \pm 3.06$ & $5.81 \pm 3.50$ & $4.16 \pm 2.91$ & $17.27^{\ddagger}$ & 0.000 & 0.273 \\
\hline & VC & $9.78 \pm 3.00$ & $5.97 \pm 3.34$ & $5.19 \pm 2.97$ & $19.98^{\ddagger}$ & 0.000 & 0.303 \\
\hline & IN & $9.31 \pm 2.43$ & $6.66 \pm 3.18$ & $6.13 \pm 3.18$ & $10.63^{\ddagger}$ & 0.000 & 0.188 \\
\hline \multirow[t]{4}{*}{ PRI } & & $94.34 \pm 12.03$ & $78.50 \pm 17.18$ & $73.65 \pm 18.18$ & $14.12^{\ddagger}$ & 0.000 & 0.235 \\
\hline & $\mathrm{BD}$ & $9.50 \pm 3.10$ & $6.59 \pm 3.78$ & $5.00 \pm 3.36$ & $14.02^{\ddagger}$ & 0.000 & 0.234 \\
\hline & MR & $8.12 \pm 2.56$ & $6.06 \pm 3.63$ & $5.74 \pm 3.59$ & $5.18^{\dagger}$ & 0.007 & 0.101 \\
\hline & VP & $9.34 \pm 2.73$ & $6.63 \pm 2.73$ & $5.81 \pm 3.25$ & $12.82^{\ddagger}$ & 0.000 & 0.218 \\
\hline \multirow[t]{3}{*}{ WMI* } & & $94.94 \pm 12.03$ & $79.63 \pm 19.71$ & $68.61 \pm 15.23$ & $21.60^{\ddagger}$ & 0.000 & 0.320 \\
\hline & DS* & $8.94 \pm 2.30$ & $6.03 \pm 4.20$ & $3.87 \pm 3.12$ & $18.75^{\ddagger}$ & 0.000 & 0.290 \\
\hline & $\mathrm{AR}^{*}$ & $8.94 \pm 2.54$ & $6.53 \pm 3.02$ & $5.06 \pm 2.52$ & $16.54^{\ddagger}$ & 0.000 & 0.264 \\
\hline \multirow[t]{3}{*}{ PSI } & & $101.72 \pm 14.58$ & $68.81 \pm 15.80$ & $62.77 \pm 13.44$ & $64.97^{\ddagger}$ & 0.000 & 0.585 \\
\hline & SS & $10.41 \pm 3.12$ & $4.10 \pm 2.78$ & $3.03 \pm 2.20$ & $67.72^{\ddagger}$ & 0.000 & 0.596 \\
\hline & $\mathrm{CD}$ & $9.66 \pm 3.10$ & $4.16 \pm 2.73$ & $3.13 \pm 2.36$ & $51.76^{\ddagger}$ & 0.000 & 0.529 \\
\hline Full-Scale IQ & & $94.72 \pm 11.89$ & $70.53 \pm 17.48$ & $62.65 \pm 15.87$ & $38.02^{\ddagger}$ & 0.000 & 0.453 \\
\hline GAI & & $94.38 \pm 12.89$ & $75.09 \pm 17.96$ & $68.68 \pm 17.66$ & $21.27^{\ddagger}$ & 0.000 & 0.316 \\
\hline CPI & & $97.22 \pm 11.47$ & $68.41 \pm 17.76$ & $58.97 \pm 15.03$ & $56.09^{\ddagger}$ & 0.000 & 0.549 \\
\hline
\end{tabular}

${ }^{*}$ means that significant univariate differences between complicated mild/moderate and severe TBI group, ${ }^{\dagger} \mathrm{p}<0.01,{ }^{ \pm} \mathrm{p}<0.001$. K-WAIS-IV: Korean Wechsler Adult Intelligence Scales-Fourth Edition, TBI: traumatic brain injury, VCI: Verbal Comprehension Index, PRI: Perceptual Reasoning Index, WMI: Working Memory Index, PSI: Processing Speed Index, BD: block design, SI: similarities, DS: digit span, MR: matrix reasoning, VC: vocabulary, AR: arithmetic, SS: symbol search, VP: visual puzzles, IN: information, CD: coding, LN: letter number sequencing, GAI: General Ability Index, CPI: Cognitive Proficiency Index 
ever, there were no significant differences in any other indexes or subtests between the two TBI groups.

\section{DISCUSSION}

In this study, patients with NCD-TBI were first classified based on the severity of their injury. Subsequently, differences between premorbid and current cognitive function were investigated and domains in which patients differed from healthy adults were identified.

The current research was conducted to account for estimated premorbid IQ among healthy controls, patients with complicated mild/moderate TBI, and patients with severe TBI. Consequently, there was no significant difference in estimated premorbid IQ among the three groups (Table 1). This was achieved as a result of the participant recruitment method, in which groups were matched for age and education level. Moreover, only three variables were used to estimate premorbid intelligence with the Korean OPIE-IV formula (raw score for the information subtest, age, and education level). Therefore, estimated premorbid IQ is presumed to be accurate, without any deviations. ${ }^{12}$ Previous studies of brain injury have also recommended a formula comprising a single WAIS-IV subtest and demographic variables, rather than multiple WAIS-IV subtests. ${ }^{18}$ Given that brain injuries tend to cause damage to entire brain regions, premorbid intelligence may be underestimated by an equation that includes multiple WAIS-IV subtests. ${ }^{22}$

A frequency analysis of the Wechsler's descriptive classification was also conducted (Table 2). Based on current IQ, about $40 \%$ of complicated mild/moderate TBI cases had a full-scale IQ of less than 70, which may indicate intellectual disability. Moreover, about $70 \%$ of severe TBI cases had an IQ less than 70. This suggests that there should be greater concern about the degree of impairment of intellectual ability in patients with severe TBI than in patients with complicated mild/moderate TBI. The probability of having normal cognitive function after TBI is $30 \%$ in complicated mild/moderate TBI and $10 \%$ in severe TBI cases.

There were significant within-group differences in estimated premorbid and current IQ for patients with both complicated mild/moderate TBI and severe TBI (Table 3). Based on the assessment of impaired cognition with the DSM-5 Assessment Measures, ${ }^{1}$ there was a difference of more than 1 standard deviation (15 points) for both TBI groups, implying that the severity of impaired cognition was more than "moderate." Based on the average current IQ classified with the WAIS, patient with complicated mild/moderate TBI may be considered to be at the "borderline" level, while patients with severe TBI may be considered to be at the "very low" level.

Table 4 presents results for the comparison of current index- es between groups. In accordance with previous studies, there were significant differences among the groups for all indexes. ${ }^{7,8}$ In terms of effect size, processing speed had the largest magnitude $\left(\eta^{2}=0.585\right)$. However, based on the results of the post-hoc test, there was an only significant difference in working memory between complicated mild/moderate and severe TBI groups.

These findings are inconsistent with previous studies. In a study by Donders and Strong ${ }^{8}$ processing speed was an indicator by which moderately severe TBI could be discriminated from less severe TBI or healthy adults. Moreover, Carlozzi et al. ${ }^{7}$ reported that only patients with severe TBI had significantly lower processing speed scores than did those with complicated mild/moderate TBI with no differences in other indexes. However, in the current study, working memory was expected to aid categorization of TBI groups based on their severity. Indeed, the most prominent feature of NCD-TBI is cognitive change, while memory dysfunction, a cardinal feature, presents after TBI. ${ }^{9}$ Working memory s a temporary, limitedcapacity memory store, which facilitates complex processes such as language, problem solving, and cognitive guidance of behavior. ${ }^{23,24}$ NCD-TBI particularly disrupts working memory, preferentially affecting memory manipulation processes rather than passive storage. Therefore, working memory impairments are thought to be related to TBI severity. ${ }^{25}$

In the current study, there was a significant difference between NCD-TBI groups in digit span and arithmetic, two subgroups subsumed within working memory. Therefore, both digit span and arithmetic are more susceptible to the effects of TBI severity than are other subtests. ${ }^{25}$ A previous study that used the Wechsler Memory Scale-IV reported that patients with severe NCD-TBI had significantly lower scores for auditory/visual/immediate/delayed memory subtests than did patients with complicated mild/moderate NCD-TBI. ${ }^{26}$

We also investigated differences in general ability and cognitive proficiency, for both of which there were significant differences among the groups. In particularly, the magnitude of between-group differences for cognitive proficiency were associated with a very high effect size $\left(\eta^{2}=0.438\right)$. This is presumably because cognitive proficiency includes subtests that belong to both working memory and processing speed domains. However, both general ability and cognitive proficiency did not demonstrate sensitivity to TBI severity.

Table 4 presents findings for comparative analyses among the groups for current subtest scores. Similar to previous studies, all the subtests differed significantly among the groups, ${ }^{7,8}$ In particular, the largest differences among groups were observed for symbol search and coding with "large" effect sizes. In the post-hoc analysis, significant differences were seen between the TBI groups for digit span and arithmetic. This con- 
tradicts previous studies, which have reported significant difference in symbol search and coding. ${ }^{7.8}$ However, our finding can be explained with similar reasoning to the above index level interpretations. Specifically, the greatest difference was observed for digit span, suggesting that both auditory rehearsal/discrimination and mental alertness/manipulation are strongly influenced by TBI severity.

The present study contributes to the understanding of the impact of NCD-TBI on cognitive function with the following main findings: 1) While previous studies have compared current IQ for of patients with NCD-TBI to those of healthy adults, the preliminary aim of this study was to compare current IQ between these groups after controlling for estimated premorbid IQ. Ensuring the same baseline IQ can aid the interpretation and comparison of current cognitive function between groups; 2) The OPIE-IV method adopted in this study is the most recent premorbid intelligence estimation protocol to be developed. In particular, the Korean OPIE-IV method used in this study is less sensitive to variations in brain injury patients and should not overestimate or underestimate premorbid intelligence; 3) Although previous studies have reported processing speed as an indicator to compare different NCD-TBI groups, our results indicate that working memory is more sensitive to TBI severity. Moreover, processing speed most accurately distinguishes TBI from healthy adults. However, among cognitive domains tested, working memory accounts for the most variance in the discrimination of NCD-TBI groups according to severity; 4) The current study also presented a comparison of general ability and cognitive proficiency among the groups. As with IQ, there was a significant difference among the groups, with a greater proportion of the variance accounted for by cognitive proficiency than by general ability.

Despite the strengths of the current study, it is subject to some limitations that could be addressed by further research. The sample size of the study is adequate for the statistical analyses performed. However, studies with larger samples should be conducted to increase the statistical power. Additionally, unlike previous studies, working memory, but not processing speed, was observed to significantly discriminate TBI according to severity. This novel finding requires replication. Lastly, this study did not employ strict criteria for the classification of NCD-TBI participants. The present study employed both loss of consciousness and neuroimaging results to stratify TBI patients. The application of all the requisite criteria was difficult because of insufficient or unreliable information provided due to the nature of emergency medicine and potentially subjective reporting by the observer. However, future studies should be undertaken with the application of at least 3-4 criteria for stratification of NCD-TBI patients based on injury severity.

\section{Acknowledgments}

This study was supported by Wonkwang University in 2019.

\section{Conflicts of Interest}

The authors have no potential conflicts of interest to disclose.

\section{Author Contributions}

Conceptualization: Sang-Yeol Lee. Data curation: Sang-Yeol Lee. Formal analysis: Min-Jung Soh, Hye-Jin Lee. Funding acquisition: Sang-Yeol Lee. Investigation: Kyu-Sic Hwang, Min-Jung Soh, Hye-Jin Lee. Methodology: Seung-Ho Jang, Kyu-Sic Hwang. Project administration: Seung-Ho Jang. Resources: Kyu-Sic Hwang, Min-Jung Soh, Hye-Jin Lee. Software: MinJung Soh, Hye-Jin Lee. Supervision: Sang-Yeol Lee. Validation: Kyu-Sic Hwang, Min-Jung Soh, Hye-Jin Lee. Visualization: Kyu-Sic Hwang, MinJung Soh, Hye-Jin Lee. Writing-original draft: Sang-Yeol Lee, Kyu-Sic Hwang. Writing_-review \& editing: Sang-Yeol Lee, Seung-Ho Jang.

\section{ORCID iDs}

Sang-Yeol Lee https://orcid.org/0000-0003-1828-9992

Kyu-Sic Hwang https://orcid.org/0000-0002-8997-8720

\section{REFERENCES}

1. American Psychiatric Association. Diganostic and Statistical Manual of Mental Disorders, Fifth Edition. Wanshington DC: American Psychiatric Association; 2013

2. Management of Concussion/mTBI Working Group. VA/DoD clinical practice guideline for management of concussion/mild traumatic brain injury. J Rehabil Res Dev 2009;46:CP1-68.

3. McDonald S, Fisher A, Flanagan S, Honan CA. Impaired perception of sincerity after severe traumatic brain injury. J Neuropsychol 2017; 11:291-304.

4. Rowley DA, Rogish M, Alexander T, Riggs KJ. Counter-intuitive moral judgement following traumatic brain injury. J Neuropsychol 2018;12: 200-215.

5. Wechsler D. Wechsler Adult Intelligence Scale-Fourth Edition (WAISIV). San Antonio, TX: Harcourt Assessment Inc.; 2008.

6. Zillmer E, Spiers M, Culbertson WC. Principles of Neuropsychology. Wadsworth; Thomson; 2008.

7. Carlozzi NE, Kirsch NL, Kisala PA, Tulsky DS. An examination of the Wechsler Adult Intelligence Scales, Fourth Edition (WAIS-IV) in individuals with complicated mild, moderate and severe traumatic brain injury (TBI). Clin Neuropsychol 2015;29:21-37.

8. Donders J, Strong CA. Clinical utility of the Wechsler Adult Intelligence Scale-Fourth Edition after traumatic brain injury. Assessment 2015;22:17-22.

9. Silver JM, McAllister TW, Yudofsky SC. Textbook of Traumatic Brain Injury. Arlington, VA: American Psychiatric Pub.; 2011.

10. Lezak MD. Neuropsychological Assessment. New York: Oxford; 2012.

11. Schoenberg MR, Lange RT, Marsh P, Saklofske DH. Premorbid Intelligence. New York: Springer; 2011.

12. Holdnack JA, Schoenberg MR, Lange RT, Iverson GL. Predicting Premorbid Ability for WAIS-IV, WMS-IV and WASI-II. WAIS-IV, WMSIV, and ACS. In: Holdnack JA, Drozdick L, Weiss LG, Iverson GL, Editors. WAIS-IV, WMS-IV, and ACS: Advanced Clinical Interpretation. New York: Academic Press, 2013, p.217-278.

13. Holdnack JA, Drozdick L, Weiss LG, Iverson GL. WAIS-IV, WMS-IV, and ACS: Advanced Clinical Interpretation. New York: Academic Press; 2013.

14. Kashluba S, Hanks RA, Casey JE, Millis SR. Neuropsychologic and functional outcome after complicated mild traumatic brain injury. Arch Phys Med Rehabil 2008;89:904-911.

15. Hwang ST, Kim JH, Park GB, Choi JY, Hong SH. Korean Wechsler Adult Intelligence Scale, Fourth Edition (K-WAIS-IV) Technical and 
Interpreetive Manual. Daegu: Korea Psychology Corporation; 2013.

16. Hwang ST, Kim JH, Park GB, Choi JY, Hong SH. Korean Wechsler Adult Intelligence Scale, Fourth Edition (K-WAIS-IV). Daegu: Korea Psychology Corporation; 2013.

17. Krull KR, Scott JG, Sherer M. Estimation of premorbid intelligence from combined performance and demographic variables. Clin Neuropsychol 1995;9:83-88.

18. Schoenberg MR, Duff K, Scott JG, Adams RL. An evaluation of the clinical utility of the OPIE-3 as an estimate of premorbid WAIS-III FSIQ. Clin Neuropsychol 2003;17:308-321.

19. Schoenberg MR, Scott JG, Duff K, Adams RL. Estimation of WAIS-III intelligence from combined performance and demographic variables: development of the OPIE-3. Clin Neuropsychol 2002;16:426-438.

20. Kim SG, Lee EH, Hwang ST, Park K, Chey J, Hong SH, et al. Estimation of K-WAIS-IV premorbid intelligence in South Korea: development of the KPIE-IV. Clin Neuropsychol 2015;29:19-29.

21. Cohen J. Statistical Power Analysis for the Behavioral Sciences. L. Hill- sdale, N.J: Erlbaum Associates; 1988.

22. Larrabee GJ, Largen JW, Levin HS. Sensitivity of age-decline resistant ("hold") WAIS subtests to Alzheimer's disease. J Clin Exp Neuropsychol 1985;7:497-504.

23. Baddeley AD, Kopelman M, Wilson BA. The Essential Handbook of Memory Disorders for Clinicians. England: John Wiley \& Sons; 2004.

24. West LK, Curtis KL, Greve KW, Bianchini KJ. Memory in traumatic brain injury: the effects of injury severity and effort on the Wechsler Memory Scale-III. J Neuropsychol 2011;5:114-125.

25. Vallat-Azouvi C, Weber T, Legrand L, Azouvi P. Working memory after severe traumatic brain injury. J Int Neuropsychol Soc 2007;13:770780.

26. Carlozzi NE, Grech J, Tulsky DS. Memory functioning in individuals with traumatic brain injury: an examination of the Wechsler Memory Scale-Fourth Edition (WMS-IV). J Clin Exp Neuropsychol 2013;35: 906-914. 